\title{
Sneezing, nasal discharge, dyspnea and sudden death in rabbits
}

\author{
Roger S. Geertsema, DVM, DACVPM, Lisa LaFranco-Scheuch, DVM, PhD, DACVP, Keith J. Jenné, DVM \& \\ Philip J. Richter, DVM, PhD, DACLAM
}

Our animal facility obtained 15 threemonth-old New Zealand Red rabbits weighing between 3.0 and $3.5 \mathrm{~kg}$ from a commercial rabbit vendor. The animals were housed individually in a room that contained other rabbits and was maintained at $21 \pm 1{ }^{\circ} \mathrm{C}$ on a 12 -h: 12 -h light: dark cycle. The rabbits received tap water and a high-fiber rabbit diet (Prolab 5P25, Brentwood, MO) fed ad libitum. The facility is fully accredited by the Association for the Assessment and Accreditation of Laboratory Animal Care, International and use of the rabbits had Institutional

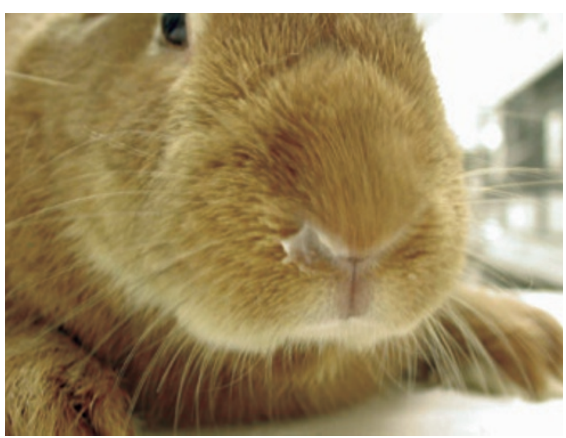

FIGURE 1 | A white, mucopurulent nasal discharge from the right nostril of a 3-month-old New Zealand Red rabbit.
Animal Care and Use Committee approval under several different study protocols.

Upon arrival, all rabbits appeared healthy with normal appetites and no sign of disease. Five days later, one rabbit was found dead. Upon examination of the other 14 rabbits, we found that one had a unilateral, white, mucopurulent nasal discharge (Fig. 1) and another was sneezing. These two rabbits had normal appetites, body temperatures and lung sounds. A third rabbit was febrile (body temperature of $40.8{ }^{\circ} \mathrm{C}$ ), seemed depressed, had not eaten the previous day's food and had labored respiration. On auscultation, lung sounds on one side were harsh with crackling.

We carried out a necropsy on the rabbit that was found dead. The thoracic cavity contained a small amount of dark red fluid and the left lung was mottled red to dark red and consolidated (Fig. 2). A fibrinous exudate covered the pleura in the dorsocaudal region of the left lung. The right side of the lung, trachea, nasal passages and tympanic bullae appeared normal. We took samples for bacterial culture from the affected lung and also from the white, mucopurulent nasal discharge (Fig. 1) of the live rabbit. Samples of lung

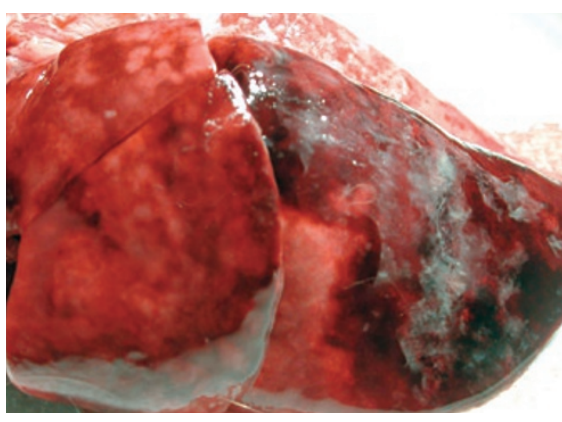

FIGURE 2 | Left side of the lungs of a 3-monthold New Zealand Red rabbit that was found dead $5 \mathrm{~d}$ after arrival in our facility. The lung lobes are dark red and consolidated. The pleura over the caudal portion are covered with multiple specks of fibrin.

were immersion-fixed in $10 \%$ neutralbuffered formalin for histopathological examination.

Based on the history, clinical signs and gross pathology, what is the likely cause of this outbreak of respiratory problems and sudden death? Would you use an antibiotic to treat the affected rabbits? If so, which antibiotic would you use?

\section{What's your diagnosis?}

